\title{
Identificación de Patrones de Movilidad Utilizando Datos en Tiempo Real Generados por Access Points en una Red de Comunicaciones de Campus. Caso de estudio: Universidad Central del Ecuador
}

Identification of Mobility Patterns Using Real-Time Data Generated by Access Point on a Campus Communications Network. Case Study: Central University of Ecuador

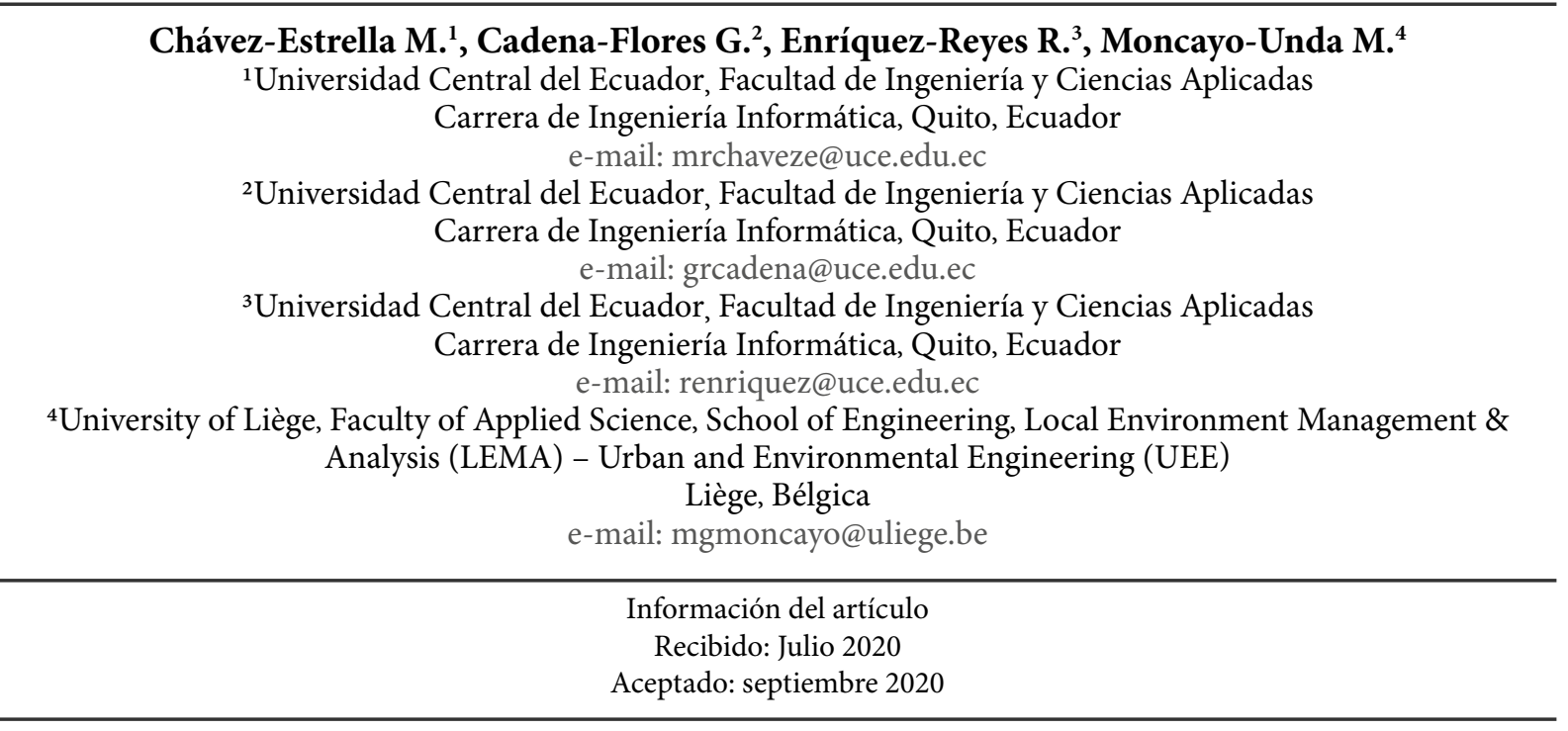

\begin{abstract}
RESUMEN
El trabajo presentado a continuación tiene como objetivo ofrecer información verídica, objetiva y en tiempo real, relacionada con patrones de movilidad generados por los usuarios en el campus, pudiendo identificarse características de los usuarios. Esto se logra mediante el uso de una plataforma informática conformada por dos aplicaciones: un API para el proceso de extracción y almacenamiento de la data depurada en la base de datos relacional, y una aplicación WEB que muestra al usuario final los resultados. Fueron utilizadas herramientas de código abierto para el desarrollo de esta plataforma. Los datos se originan en los access points ubicados en la red de comunicaciones del campus, y esta data obtenida es anonimizada y depurada para su análisis. La plataforma ha sido desplegada en un contexto universitario, específicamente en la Universidad Central del Ecuador, para ser utilizada como caso de estudio.
\end{abstract}

Palabras clave: aplicación WEB, API, tecnología, patrones de movilidad, big data, tiempo real.

\begin{abstract}
The work presented below aims to provide truthful, objective and real-time information, pertaining to mobility patterns generated by users on campus, being able to identify user characteristics, this is achieved through the use of a computer platform made up of two applications: an API for the process of extracting and storing the purified data in the relational database, and a web application that shows the results at the end. Open source tools were used for the development of this platform. The data originates from the access points located in the campus communication network, and this data obtained is anonymized and refined for analysis. The platform has been deployed in a university context, specifically at the Central University of Ecuador, to be used as a case study.
\end{abstract}

Keywords: WEB application, API, technology, mobility patterns, big data, real time. 


\section{Introducción}

El continuo desarrollo de la tecnología, juntamente con las redes móviles y su acceso a internet generan nuevas oportunidades para el estudio de patrones de movilidad. Cabe mencionar en este contexto, el estudio realizado en un campus canadiense [1], en donde se logró con éxito identificar la localización temporal y espacial de actividades mediante la utilización de big data. Sin embargo, en el estudio mencionado se presentan correcciones necesarias mediante la aplicación de métodos estadísticos para sesgar los datos. Como complemento del estudio se corrobora la riqueza informativa de los datos que se obtienen al emplear encuestas y otros métodos tradicionales de levantamiento en campo, pero más importante, en los resultados están presentes datos de la dimensión social [1].

En el mismo ámbito del artículo se presentó otro estudio basado en Dhaka, en el cual se emplea un análisis similar a través de una red de dispositivos móviles. En este estudio se realiza la comparación del método de levantamiento en campo denominado matriz de origen destino (OD) mediante puntos fijos. Se demostró que los modos tradicionales para llenar las matrices (OD) son apreciados como una alta inversión económica y un largo periodo de elaboración, a diferencia de los métodos de dispositivos móviles en una red de internet [2].

La aplicación de tecnologías digitales desde dispositivos móviles, como son el geoposicionamiento global y sistemas de información geográfica, ha dado paso a aportes característicos en estudios relacionados a la movilidad. La obtención de datos, anteriormente realizada a través de la observación de entidades en el sitio, ya sean éstas personas, automóviles u otras, hoy son remplazadas por sistemas de datos colectados en tiempo real, con una ubicación cada vez más precisa.

Además de lo mencionado anteriormente, consideramos la posibilidad de ahondar en los datos recolectados, haciendo posible la vinculación de atributos específicos a las entidades, como identificación de los dispositivos móviles, el tipo de dispositivo, el tiempo de conexión, el punto del registro e inclusive las trazas de movilidad, mediante varios dispositivos tanto fijos, proveedores de la red de datos (tal es el caso de los access points-APs), como móviles, en el caso de los receptores y usuarios de esta red (teléfonos inteligentes, tablets, etc.).

Se conoce evidencia actual sobre la adquisición de información de patrones de movilidad mediante puntos fijos, la cual ha sido procesada y ha otorgado excelentes resultados capaces de ser aplicados en estudios similares. Tal es el caso del estudio de [3], quienes lograron con gran precisión modelar la movilidad a partir de puntos wifi, comprendiendo así la segmentación de la población localizada. Otro estudio efectuado por [4], realiza una técnica similar desde la extracción de información donde se incluye perfiles de usuarios y clasificación de los dispositivos tecnológicos, con un costo relativamente bajo.

Otros estudios inclusive han llegado a plantear modelos predictivos de ocupación del espacio, como es el caso de [5] y de simulación de la movilidad humana, planteando regularidad del comportamiento espacio temporal de los usuarios de dispositivos móviles, como es el caso de [6].

La estructura del artículo está dividida en 4 apartados. El primero de éstos es la Introducción, encargada de poner en contexto la presentación de este artículo, basado en publicaciones que están relacionadas con el estudio de patrones de movilidad. El segundo apartado es la Fundamentación, en la cual se describe un concepto sólido del artículo, basado en ideas que están vinculadas con el desarrollo de la plataforma de movilidad. Un tercer apartado abarca los Resultados y discusión, en donde se presentan los casos de estudios en el campus universitario, mediante la aplicación de ciertos casos de consulta, explicando los parámetros válidos para cada uno de éstos. El cuarto y último apartado son las Conclusiones, en donde se incluye los trabajos futuros.

\section{Fundamentación}

Con la finalidad de generar nuevos métodos para estudios de patrones de movilidad se propone desarrollar una plataforma informática capaz de recopilar información obtenida desde los access points, ubicados en la red inalámbrica del campus, y generar una aplicación WEB que entregue una visualización de los patrones de movimiento en tiempo real mediante una interfaz amigable con el usuario, para así poder tomar decisiones basadas en datos [7]. 
La plataforma de movilidad está construida con datos que entregarán una importante cantidad de información para futuros estudios estadísticos, planificaciones y análisis de movilidad, debido a que éstos almacenan características principales de cada usuario en el campus, intervalos de conexión a la red e información sobre los dispositivos desde donde se realizaron las conexiones [7].

Para lograr este fin, se ha seleccionado a la Universidad Central del Ecuador como caso de estudio para obtener sus patrones de movilidad; de esta forma, miembros de la comunidad, interesados en conocer estos patrones para estudios o mejora de servicios, podrán acceder a ellos de una manera sencilla, verídica y confidencial [7]. El campus universitario ha tenido varios inconvenientes relacionados con la infraestructura arquitectónica original de la universidad, el uso que se hace de la misma y el acceso a internet dentro del campus. Entre estos inconvenientes, se presentan los más relevantes:

- Accesos peatonales (puertas) cerrados por motivos de seguridad, lo que causa que se elijan otras rutas que a veces complican la movilidad dentro del campus universitario.

- Ubicación de los access points en lugares incorrectos, lo que causa que varios de éstos se saturen por muchas conexiones, al igual que otros no sean usados a su capacidad total o que casi no se usen.

- Áreas verdes de la ciudadela universitaria con apariencia desagradable. Esto a causa de los senderos creados por los estudiantes ya que les facilita su movilidad.

- Robos en determinados lugares donde no existe personal de seguridad, ya que las autori- dades no tienen una visión real de cuántos estudiantes se conectan y su lugar de conexión; por esta razón la seguridad es escasa en zonas específicas, sin un análisis previo, lo que dificulta la llegada a ciertos lugares donde ocurren los percances.

Los datos que servirán para la construcción de la plataforma de movilidad otorgan gran cantidad de información útil para futuras planificaciones, estudios estadísticos y análisis de movilidad, pues almacenan las características principales de cada individuo, intervalos de tiempo e información sobre los dispositivos que realizaron la conexión. Nos permite definir diferencias entre métodos tradicionales y los obtenidos con la ayuda de tecnología, que es el aporte del trabajo.

\section{Metodología}

La investigación propone un método para identificar de forma gráfica las correlaciones entre variables de tipo métrico, cualitativo y espacial. Para la presentación de este método se creó una plataforma de movilidad constituida por dos componentes. El primer componente, denominado API (interfaz de programación de aplicaciones), se encarga de obtener la información, depurarla, clasificarla y almacenarla en la base de datos; el segundo componente, es una aplicación WEB que permite mostrar de una forma gráfica la información que se obtiene al someter a un proceso de análisis los datos recolectados por el API.

La plataforma de movilidad utiliza varias herramientas de desarrollo de software, tanto para frontend como para backend. En la siguiente tabla se muestran los detalles de cada una de las herramientas utilizadas para el desarrollo de la aplicación: 
Tabla 1. Herramientas usadas para la realización de la plataforma de movilidad [7].

\begin{tabular}{lll}
\hline Nombre & Descripción & Versión \\
\hline Windows & Sistema operativo & 10 \\
\hline Java jdk & Kit de desarrollo Java & 1.8 .0 \\
\hline Java jre & Java runtime environment & 1.8 .0 \\
\hline Spring framework & Framework que usa Java & 5.1 .9 \\
\hline Spring tool suite & IDE de desarrollo & 3.9 .8 \\
\hline Spring boot & Autoconfiguración proyectos Spring & 2.1 .8 \\
\hline PostgressQL 10 & Motor de base de datos & 10.10 \\
\hline pgAdmin 4 & IDE de administrador & 4.13 \\
\hline Apache Tomcat 8.5 & Contenedor de servlets & 8.5 \\
\hline QGIS & Sistema de información geográfica & 2.8 .8 \\
\hline QGIS2WEB & Complemento de QGIs & 2 \\
\hline Apache csv & Librería que trabaja con archivos csv & 1.5 \\
\hline Thymeleaf extras & Librería que permite trabajar sobre Frontend & 3.0 .2 \\
\hline Apache BeanUtils & Librería de arquitectura basada componentes & 1.9 .3 \\
\hline IText Core & Librería para crear y manipular PDF & 5.5 \\
\hline JQuery & Interactuar con los documentos HTML & 3.4 .1 \\
\hline HTML5 & Estructurar y presentar el contenido & 5 \\
\hline Css3 & Lenguaje de diseño gráfico para HTML & 4 \\
\hline
\end{tabular}

En la siguiente imagen se muestra la arquitectura de la plataforma de movilidad desde una perspectiva técnica, tomando como inicio la recolección de la data desde los access points del campus en estudio, hasta la presentación de los patrones de movilidad al usuario, luego de haber sido estos datos depurados, clasificados e interpretados en la aplicación wEB. 


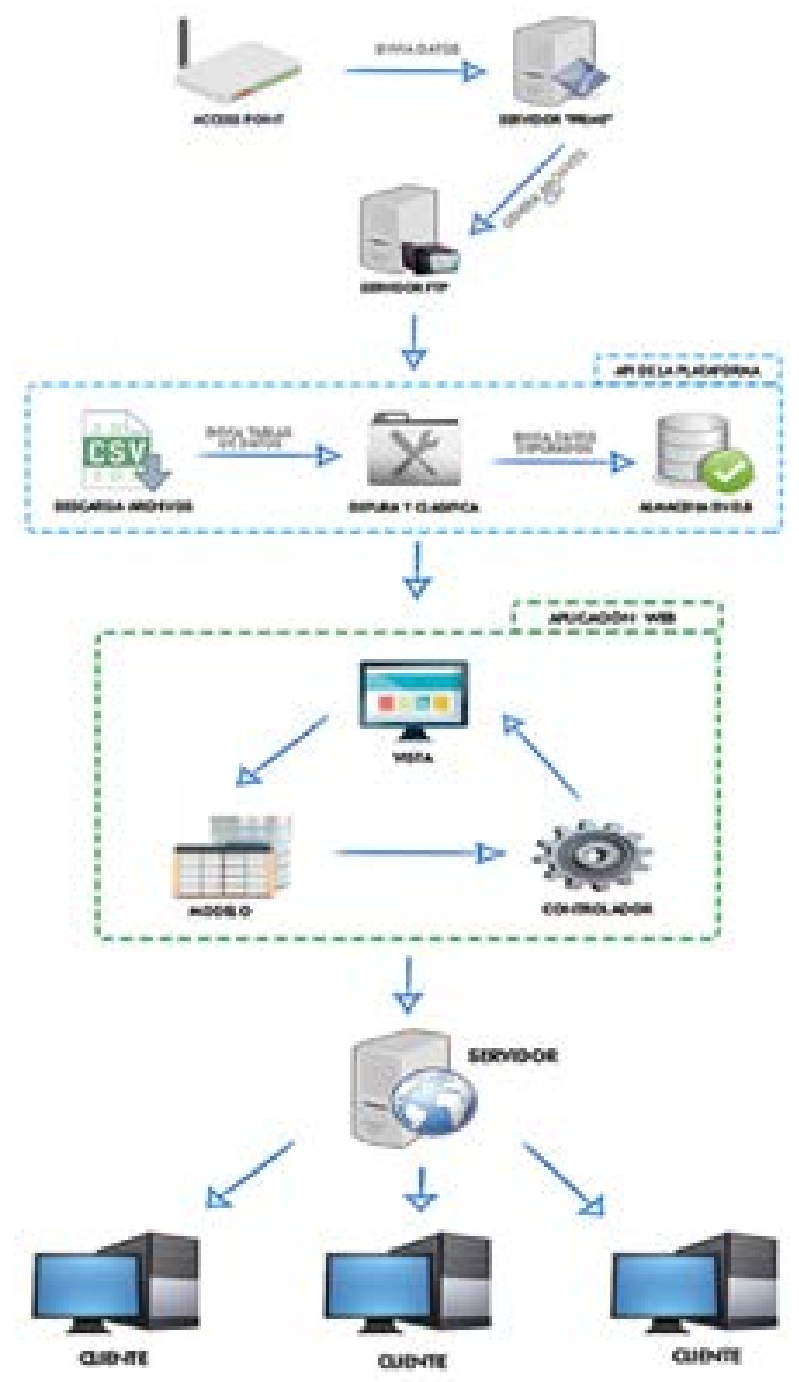

Figura 1. Arquitectura de la plataforma de movilidad.

El primer paso dentro de esta arquitectura es obtener los datos de todas las conexiones que se realizan a la red inalámbrica de la universidad. Esta tarea la realiza un servidor alojado en el data center de la universidad que recoge la información recolectada por cada uno de los access points ubicados en el campus principal y la almacena en un servidor FTP como archivos con extensión .csv para su posterior tratamiento y uso.

En esta etapa del proceso entra en ejecución la API; esta API se ejecutará como un proceso adicional del servidor en el cual está instalada la aplicación, ya que siempre va a estar ejecutándose y encargán- dose de copiar datos de los archivos que se encuentran en el servidor FTP; todos estos datos pasarán por un proceso de depuración, validación, clasificación y almacenamiento.

- Depuración: Los archivos son generados con un encabezado especificando todo lo que contiene el archivo, este encabezado es eliminado ya que no forma parte de los datos de interés para la plataforma.

- Validación: Se verifica que los diferentes registros tengan todos los campos, que no existan campos vacíos y, además, cada campo no tenga caracteres especiales. 
- Clasificación: Se clasifica todos los datos de acuerdo con el siguiente modelo entidad relación:

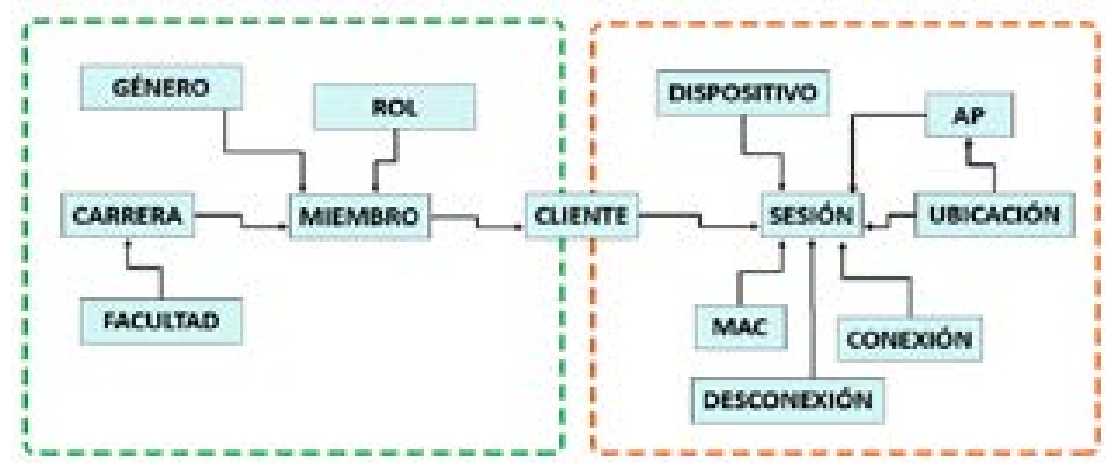

Figura 2. Modelo entidad relación que utiliza la plataforma de movilidad.

En el modelo de la figura 2 se muestran dos conjuntos de datos clasificados. El primer conjunto de datos está constituido por las tablas género, rol, facultad, carrera, miembro. Todas estas tablas contienen información de los individuos que conforman la comunidad universitaria, mientras que el segundo conjunto de datos constituido por las tablas cliente, dispositivo, sesión, AP, ubicación, MAC, conexión, desconexión, representan los datos que se obtienen de las conexiones, precisamente los datos que se encarga de almacenar el API.

- Almacenamiento: Con todos los pasos aplicados satisfactoriamente, el siguiente paso consiste en el almacenamiento de los datos dentro de una base de datos, en este caso se usó la base de datos relacional PostgresQL.

Con todos los datos consolidados en la base de datos, ya pueden ser usados por la aplicación WEB que permite mostrar de forma gráfica los resultados del proceso y análisis de estos datos.

\section{Resultados y discusión}

Los mapas que presenta la aplicación son graficados con base en filtros que el usuario debe elegir previamente antes de que se grafiquen. Entre estos filtros se encuentran los siguientes:

- Filtro del tiempo:

- mes, semana, día y hora

- Filtro de género:

Masculino, femenino e invitado (los invitados son personas no pertenecientes a la comunidad universitaria que se conectan a los access points para hacer uso del internet).
- Filtro de rol:

- estudiantes, docentes, servicios e invitados.

- Filtro de lugar:

Se debe elegir la facultad de acuerdo con lo que se quiere buscar. Una vez que se escoja la facultad se puede elegir una carrera o todas las carreras de la facultad seleccionada.

El software desarrollado proporciona la funcionalidad de obtener datos estadísticos en tiempo real o histórico de patrones de movilidad, estos datos pueden ser consultados mediante 4 filtros principales, estos son:

- Según el rol: estudiante, docente y administrativo

- Filtro de género: masculino y femenino

- Filtro de ubicación: departamento, piso y nombre del access point

- Filtro de origen: miembros pertenecientes a cierta facultad y cierta carrera

Los datos pertenecientes a los miembros de la comunidad universitaria han sido sometidos a filtros de anonimización, dando solo resultados estadísticos sin información personal, de acuerdo con la Ley de Protección de Datos que fue entregada a la Asamblea Nacional en busca de su aprobación.

Después de algunas consultas hemos podido observar patrones de movilidad que permiten a los investigadores elaborar modelos con más información que los métodos tradicionales con encuestas 
y con costos significativamente menores. A continuación, mostramos resultados relevantes de las consultas, en función de su cantidad y precisión que sería muy costoso realizarlo con encuestas:

\section{Movilidad de estudiantes de cultura física}

Mediante una consulta a la aplicación, con datos relacionados a los estudiantes de la Facultad de Cultura Física, se puede mostrar un mapa con to- dos los recorridos dentro del campus principal que han realizado los estudiantes de esta facultad. Para este caso se eligió la gráfica de los recorridos en un intervalo de tiempo de un mes, dando como resultado el siguiente mapa [7]:

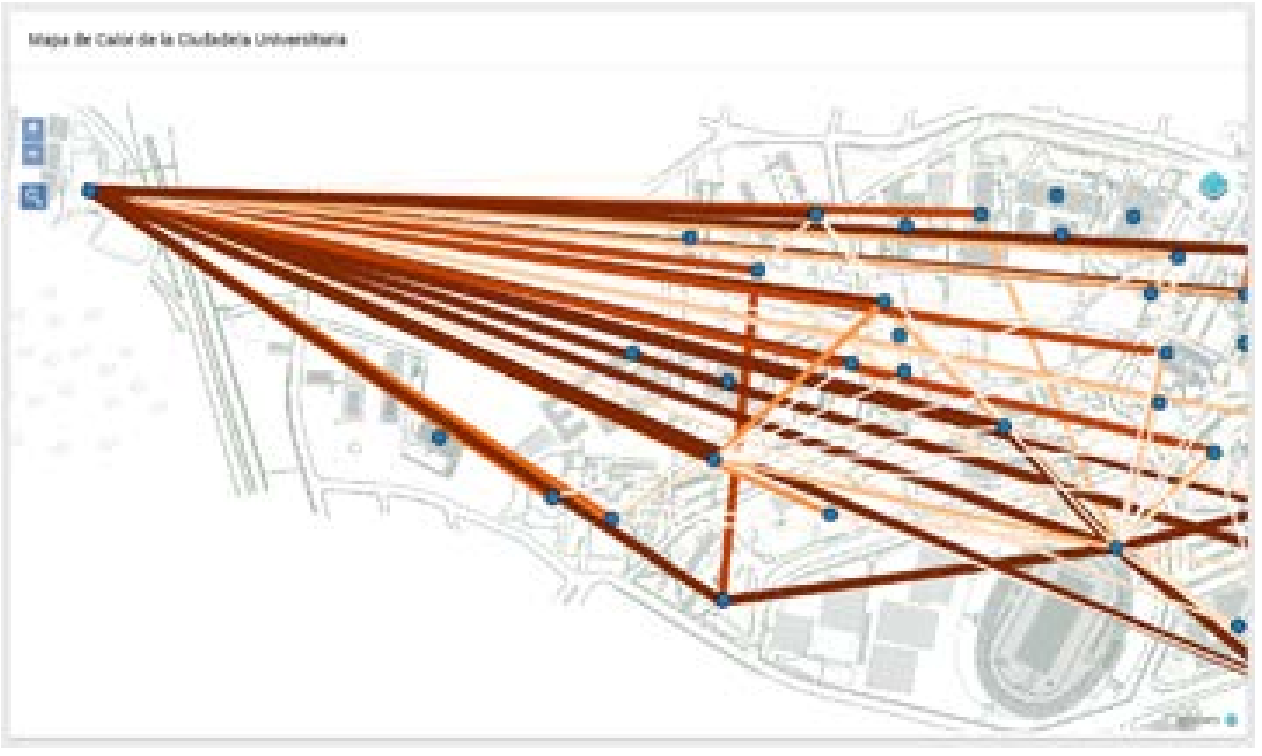

Figura 2. Mapa de movilidad de los estudiantes de la Facultad de Cultura Física [7].

Este mapa genera una tabla de datos, para entender su interpretación, la cual se muestra a continuación:

Tabla 2. Datos del mapa de movilidad de los estudiantes de la Facultad de Cultura Física [7].

\begin{tabular}{lll}
\hline Origen & Destino & N. ${ }^{\circ}$ de usuarios \\
\hline Estadio & Facultad Cultura Física & 976 \\
\hline Facultad Cultura Física & Estadio & 952 \\
\hline Edificio de Idiomas & Facultad Cultura Física & 388 \\
\hline Facultad Cultura Física & Edificio de Idiomas & 323 \\
\hline Facultad Cultura Física & Biblioteca & 269 \\
\hline
\end{tabular}

El informe presenta una participación de más de 3000 personas que recorrieron las vías, lo que significa que usando los métodos tradicionales se necesitaría muchos encuestadores durante el mismo tiempo de muestra, impactando en el presupuesto de los proyectos de movilidad en un campus, tomando en cuenta que sería muy complejo físicamente determinar a qué facultad pertenecen; con el software se puede granular la información de acuerdo con la estructura de la base de datos. 


\section{Concentración de invitados en el campus}

La figura 3 muestra las conexiones que realizan los invitados, la cual se puede obtener al realizar una consulta con los datos de las personas que se conectan a la red de invitados del campus [7].

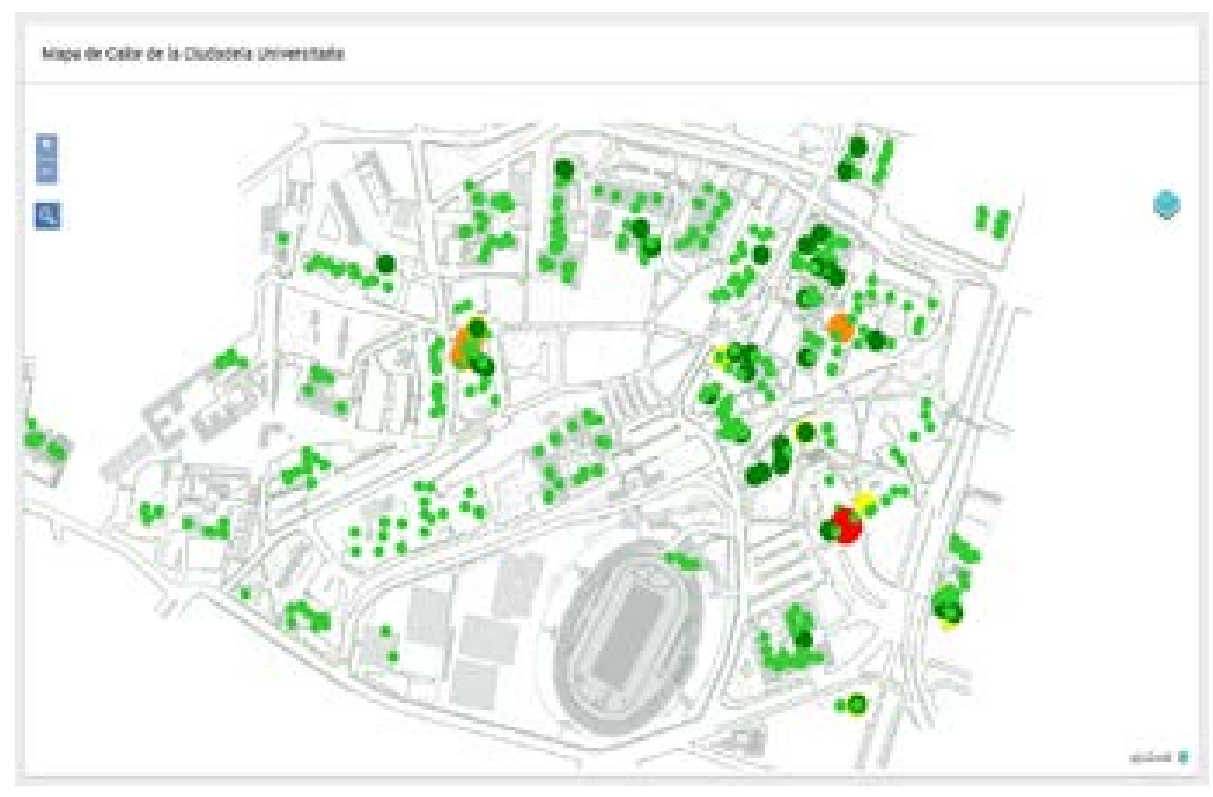

Figura 3. Mapa de calor de los invitados en la ciudadela universitaria.

Al visualizar el mapa de la ciudadela universitaria, expresado como mapa de calor, se puede ver que existen 3 lugares que concentran las conexiones de usuarios que se conectaron a la red de invitados; para comprender de una manera más fácil lo que representa el mapa también existe una tabla la cual presenta datos relacionados a las conexiones que se hacen a cada access point de la ciudadela universitaria [7]:

Tabla 3. Datos del mapa de calor de los invitados en la ciudadela universitaria

\begin{tabular}{lll}
\hline Origen & Destino & N. ${ }^{\circ}$ de usuarios \\
\hline Dirección General Financiera & Administración-Teatro & 9567 \\
\hline Biblioteca General & Administración Central & 6575 \\
\hline Derecho & Facultad de Jurisprudencia & 6245 \\
\hline Biblioteca General & Administración Central Interior & 6194 \\
\hline Economía Edificio A & Facultad de Economía Biblioteca & 5610 \\
\hline
\end{tabular}

Haciendo una comparación con los métodos tradicionales vemos que sería muy costoso sacar este patrón, pues se necesitaría encuestadores durante periodos largos de tiempo, además la precisión de los datos no se podría comparar con los obtenidos con la tecnología en tiempo real. 
Lugares de conexión de los estudiantes de ciencias médicas

Una de las opciones de la aplicación permite visualizar en tiempo real o en una fecha específica las estadísticas de las conexiones dentro del campus universitario. Para definir el patrón se utilizaron los datos de los estudiantes de la Facultad de Ciencias Médicas, dando como resultado el siguiente gráfico [7]:

\section{0 \\ Lugares de Conexión}

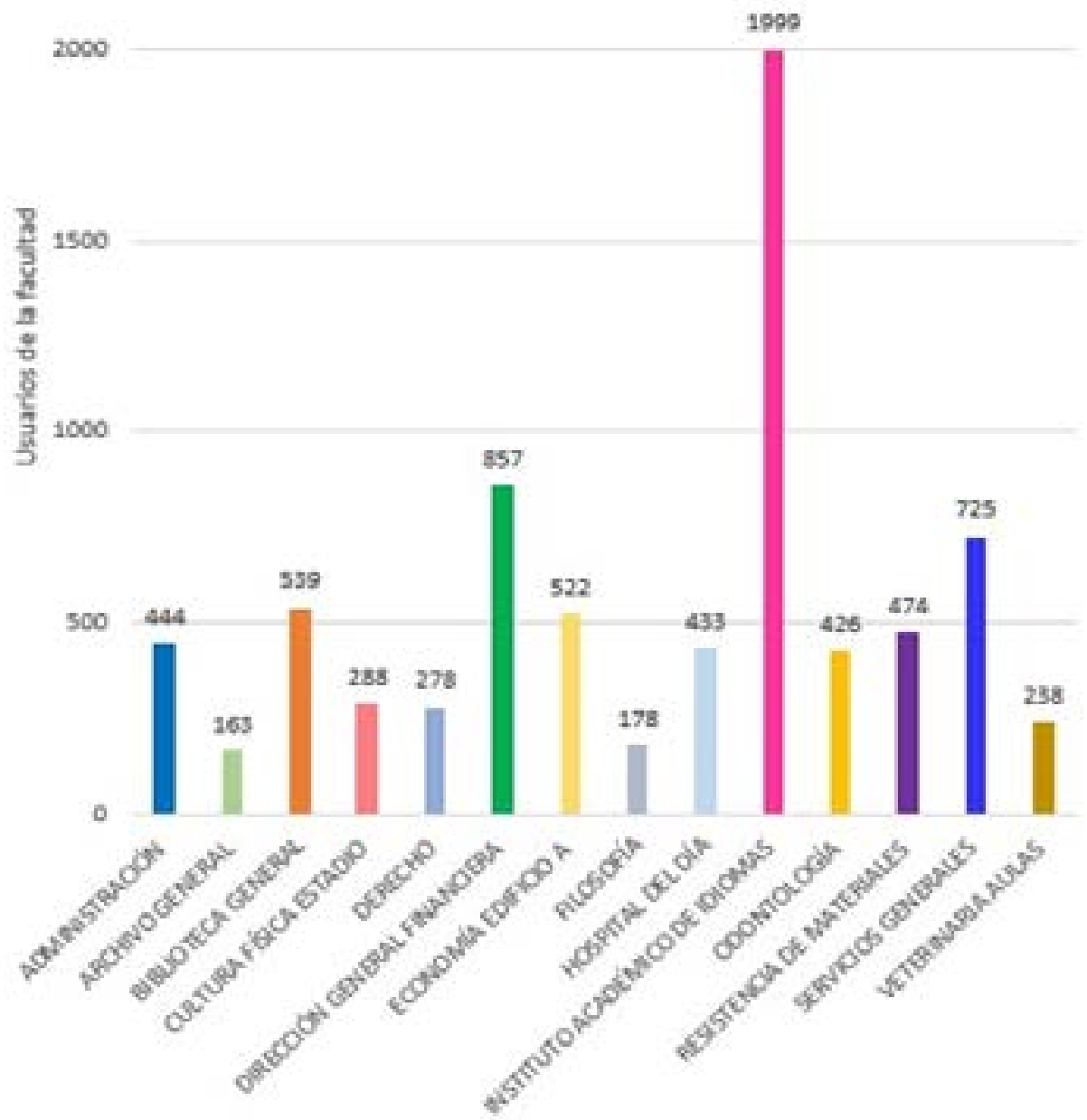

Figura 4. Gráfico de barras sobre los lugares de conexión de estudiantes de Ciencias Médicas [7].

Se puede visualizar que el lugar en donde existen más conexiones es el Instituto de Idiomas, después le siguen la Dirección General Financiera y el Departamento de Servicios Generales [7].

El obtener este nivel de granularidad en los datos es muy costoso por métodos tradicionales. Los resultados obtenidos pueden impactar incluso en las decisiones estratégicas de la universidad para crear centros satélites con el fin de evitar que alumnos alejados de los edificios de servicios administrativos gasten tiempo y dinero para llegar a sus clases en otro campus.

Si observamos el mapa de calor generado con la misma consulta, se verifica los datos anteriormente expresados: 


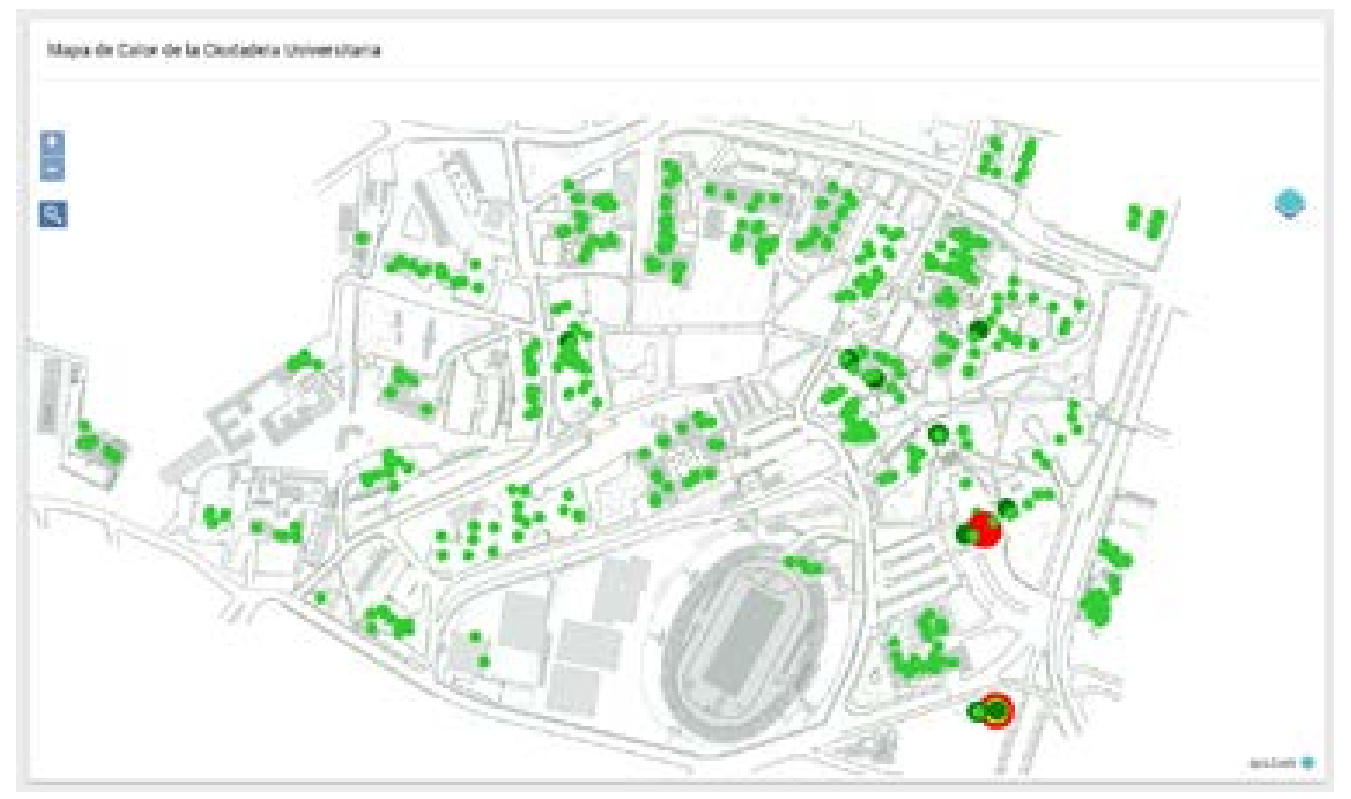

Figura 5. Mapa de calor de los estudiantes de la Facultad De Ciencias Médicas en la ciudadela universitaria [7].

La información que presenta la aplicación muestra el potencial que posee el software desarrollado, al utilizar los access points como elementos para incrementar el nivel de integridad y veracidad de los datos. Con el software se puede obtener información relevante sobre los patrones de movilidad que se producen en el campus central, tales como recorridos realizados, lugares con mayor frecuencia de conexiones, estadísticas de dónde se conectan las personas con los diferentes roles que les asigna la red, entre otros [7].

La información que entrega la plataforma permite conocer los lugares que más frecuentan los integrantes de la ciudadela universitaria. Ha permitido identificar cuáles son los recorridos de grupos de usuarios, detallados por género, rol, facultad de procedencia, entre otras; entregar estadísticas de clasificación de usuarios y dispositivos de conexión, todo esto en tiempo real o histórico, para su posterior análisis [7].

Observamos también que los datos provenientes de los access points permiten analizar información sobre los miembros de la comunidad universitaria, lo que facilita caracterizarlos por género, rol, edad, facultad de pertenencia o lugar de trabajo que incluye dispositivo de conexión, esto permite a la aplicación WEB mostrar estadísticas útiles en estudios de movilidad [7].

\section{Conclusiones}

El uso de tecnologías de adquisición de datos en tiempo real y la utilización de análisis de big data permite mejorar los métodos tradicionales en función de tiempo y costo.

La arquitectura que se utilizó en el desarrollo de la plataforma de movilidad permite alcanzar los objetivos de los investigadores y la aplicación de tecnologías nuevas como ágil por los desarrolladores, demostrando que es posible la creación de proyectos multidisciplinarios con éxito.

Se puede pensar en la posibilidad de la fusión de la plataforma de movilidad con un sistema de seguridad institucional, en el cual la plataforma contaría con herramientas propias para alertar de posibles sucesos en contra de la integridad de los miembros de la comunidad universitaria.

\section{Referencias}

[1] G. Poucin, B. Farooq y Z. Patterson, "Activity patterns mining in Wi-Fi access point logs", Computers, Environment and Urban Systems, vol. 67, pp. 55-67, 2018. 
[2] C. Choudhury, S. Iqbal, P. Wang y M. González, "Development of origin-destination matrices using mobile phone call data., Transportation Research Part C: Emerging Technologies, vol. 40, pp. 6374, 2014.

[3] M. Traunmueller, N. Johnson, A. Malik y C. Kontokosta, "Digital footprints: Using WiFi probe and locational data to analyze human mobility trajectories in cities., Computers, Environment and Urban Systems, vol. 72, pp. 4-12, 2018.

[4] A. Redondi y M. Cesana, "Building up knowledge through passive WiFi probes.", Computer Communications, vol. 117, pp. $1-12,2018$.

[5] W. Wang, J. Chen, T. Hong y N. Zhu, "Occupancy prediction through Markov based feedback recurrent neural network (M-FRNN) algorithm with WiFi probe technology", Building and Environment, vol. 138, pp. 160-170, 2018.
[6] K. Keramat Jahromi, M. Zignani, S. Gaito y P. G. Rossi, "Simulating human mobility patterns in urban areas", Simulation Modelling Practice and Theory, vol. 62, pp. 137-156, 2015.

[7] M. Chávez, R. Enríquez y G. Cadena, "Desarrollo de una plataforma informática para identificar patrones de movilidad en la Universidad Central del Ecuador mediante los datos en tiempo real generados por los access point ubicados en la Ciudadela Universitaria", tesis pregrado, Universidad Central del Ecuador, Quito, Ecuador, 2020. 